\title{
Frame-shift Mutation Causes the Function Loss of TaMYB-A1 Regulating Anthocyanin Biosynthesis in Triticum aestivum
}

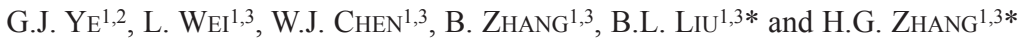 \\ ${ }^{1}$ Key Laboratory of Adaptation and Evolution of Plateau Biota (AEPB), Northwest Institute of Plateau \\ Biology, Chinese Academy of Sciences, Xining 810008, China \\ ${ }^{2}$ University of Chinese Academy of Sciences, Beijing 100049, China \\ ${ }^{3}$ Qinghai Province Key Laboratory of Crop Molecular Breeding, Xining 810008, China
}

(Received 25 February 2016; Accepted 5 July 2016)

\begin{abstract}
Red coleoptile is an easily observed trait in Triticum aestivum and can provide some protection against stress. Here, TaMYB-A1 or TuMYB-A1, homologous to TaMYB-D1, which controls red coleoptile formation in the common wheat cultivar ' $G y 115$ ', was isolated from eight T. aestivum and 34 T. urartu cultivars. The genome sequence of TaMYB-A1 was 867 bp with an intron of $93 \mathrm{bp}$, which was similar to the MYBs regulating anthocyanin biosynthesis in T. aestivum but different from other MYB transcription factors regulating anthocyanin biosynthesis. TaMYB-A1 had an integrated DNA-binding domain of 102 amino acids and a transcriptional domain of 42 amino acids, which was responsible for regulating anthocyanin biosynthesis. TaMYB-A1 was assigned to the same branch as the MYBs regulating anthocyanin biosynthesis in a phylogenetic tree. A transient expression analysis showed that TaMYB-A1 induced 'Opata' coleoptile cells to synthesize anthocyanin with the help of ZmR. A non-functional allele of TaMYB-al existed in common wheat cultivars containing $r c-a 1$. One single nucleotide was deleted 715 bp after the start codon in TaMYB-al compared with TaMYB-A1. The deletion caused a frame shift mutation, destroyed the DNA transcription activator domain, and resulted in TaMYB-al losing its ability to regulate anthocyanin biosynthesis in 'Opata' coleoptile cells. Those cultivars with functional TaMYB-A1 or $T u M Y B-A 1$ have red coleoptiles. The isolation of TaMYB-A1 should aid in understanding the molecular mechanisms of coleoptile traits in T. aestivum.
\end{abstract}

Keywords: Triticum aestivum, red coleoptile, MYB transcription factor, function verification

\section{Introduction}

Red coleoptile is an easily observed trait in Triticum aestivum that occurs when anthocyanin accumulates in the tissue. Anthocyanin endows the coleoptile with the red colour, resulting in a good phenotypic marker for differentiating cultivars (Bleiss and Ludwig 1990). Anthocyanin is also an important secondary metabolite in plants, protecting them from damage caused by strong light, heavy metals, and pathogens (Ahmed et al. 2015;

\footnotetext{
*Corresponding authors; E-mails: blliu@nwipb.cas.cn; hgzhang@nwipb.ac.cn
} 
de Oliveira et al. 2015; Ilk et al. 2015). Coleoptiles usually turn red under strong light conditions and other stresses (Lawson and Weintraub 1975; Zhang et al. 2014).

The anthocyanin biosynthesis pathway is a metabolic pathway relatively clearly understood in plant molecular genetics. The main structural genes of anthocyanin biosynthesis encode phenylalanine ammonia-lyase, chalcone synthase, chalcone isomerase, favanone 3-hydroxylase, flavonoid 3'-hydroxylase, dihydroflavonol 4-reductase, leucoanthocyanidin dioxygenase, and flavonoid 3-O-glucosyltransferase (Pourcel et al. 2012; Kayesh et al. 2013). The structural genes are regulated mainly by the MYB-bHLH-WD40 (MBW) complex (Xie et al. 2011). Usually, the overexpression of the MYB and $b H L H$ genes can induce the expression of the structural genes and anthocyanin biosynthesis because of the background expression of WD40 is enough for the formation of the MBW complex in plant (Gonzali et al. 2009; Zhang et al. 2013). The inactivation of any gene can block the whole metabolic pathway, causing a pale plant tissue phenotype. The allelic variations of the $M Y B$ genes more commonly cause colour differentiation in plants. DNA sequence variation within the promoter of functional $V v m y b A 1$ is associated with the flesh pigmentation content of intensely coloured grape varieties. Similar functional variations have also been observed for Arabidopsis AtMYB75 and AtMYB90 (Borevitz et al. 2000), petunia $A N 2$ (Quattrocchio et al. 1999), and sweet potato MYB1 (Mano et al. 2007). In monocotyledons, the famous $M Y B$ regulator $Z m C 1$, related to the discovery of transposons, regulates anthocyanin biosynthesis in maize (McClintock 1950). For T. aestivum, a 19 bp deletion in the coding sequence of TaMYB10-B is responsible for the white grain trait (Himi et al. 2011a).

The classic genetics of the red coleoptile trait have been studied for several years in common wheat. The anthocyanin pigmentation of wheat coleoptiles is controlled by $R c$ genes $(R c-A 1, R c-B 1$, and $R c-D 1$, on the short arms of chromosomes $7 \mathrm{~A}, 7 \mathrm{~B}$, and $7 \mathrm{D}$, respectively) (Khlestkina et al. 2002). Wheat cultivars carrying any one of these three genes have red coleoptiles. For example, the wheat cultivar 'Hope' $(R c-A 1 R c-B 1 r c-d 1)$ shows intense coleoptile anthocyanin pigmentation, while ' $C S$ ' ( $r c-a 1 r c-b 1 r c-d 1)$ shows no pigmentation (uncoloured coleoptile) (Himi et al. 2005). The substitution lines ' $\mathrm{CS}$ ' ('Hope' 7A) (Rc-A1rc-b1rc-d1), 'CS' ('Hope' 7B) (rc-alRc-B1rc-d1), and 'CS' (Aegilops tauschii 7D) (rc-alrc-b1Rc-D1) also show red coleoptiles (Himi et al. 2011b; Khlestkina et al. 2008). After analysing the expression levels of structural genes in the coleoptiles of the above substitution lines, each dominant $R c$ allele was hypothesized to encode a transcriptional activator (Ahmed et al. 2006). Recently, the candidate dominant $R c$ alleles were isolated from the short arms of chromosomes 7A, 7B, and 7D (Himi and Taketa 2015), and the candidate dominant TaMYB-Dl on the 7D chromosome was linked to the red coleoptile trait (Wang et al. 2015). In this article, TaMYB-A1 and the corresponding loss-of-function allele TaMYB-a1 at chromosome 7A was isolated from T. aestivum and T. urartu, and their functions in regulating anthocyanin biosynthesis were verified. 


\section{Materials and Methods}

\section{Plant materials}

In total, 236 T. urartu cultivars and $14 T$. monococcum cultivars were collected from the SDA-ARS National Small Grains Collection. In addition, 66 common wheat (T. aestivum L., $2 n=6 x=42$, AABBDD) cultivars planted widely in Qinghai Province were collected from the Chinese Crop Germplasm Resources (Xining, China). The common wheat cultivars 'CS' and 'Opata' were stored at the Northwest Institute of Plateau Biology, Chinese Academy of Sciences (Xining, China) (Table S1*). The coleoptile phenotype was checked following the procedure: 10 seeds were soaked in $70 \%$ ethanol for $1 \mathrm{~min}$, surface-sterilized in 3\% sodium hypochlorite for $20 \mathrm{~min}$, and then rinsed five times with sterile water. Sterilized seeds were placed on two layers of filter paper moistened with $6 \mathrm{ml}$ of sterile water in a Petri dish and incubated at $4{ }^{\circ} \mathrm{C}$ in the dark for $24 \mathrm{~h}$ to synchronize germination. These seeds were transferred to a growth chamber for germination at $24{ }^{\circ} \mathrm{C}$ under continuous white light $\left(60 \mu \mathrm{mol} \mathrm{m}^{-2} \mathrm{~s}^{-1}\right)$ for $4 \mathrm{~d}$, and then their phenotypes were recorded.

\section{Genomic DNA, total $R N A$, and $c D N A$ preparation}

DNA was isolated from $1 \mathrm{~g}$ of 10-day-old seedling coleoptiles according to the methods of Yan et al. (2002). Total RNA was extracted from $\sim 0.5 \mathrm{~g}$ of coleoptiles with the Tiangen RNA prep Pure Plant Kit (Tiangen Company, Beijing, China). cDNA was obtained from total RNA using the Thermo Revert Aid First Strand cDNA Synthesis Kit (ThermoFisher Scientific, Shanghai, China).

\section{Gene isolation}

The coding sequences of TaMYB-D1 were queried, using the BLAST algorithm, against the genomic sequence database of common wheat (AABBDD) (International Wheat Genome Sequencing Consortium. 2014). The contig gnl_IWGSC_7AS_IWGSC_chr7AS ab_k71_contigs_longer than_200_4108742 homologous to TaMYB-D1 is present on the short arm of the 7A chromosome. Scaffold101589 was found in the T. urartu (progenitor of the wheat A genome) (http://gigadb.org/dataset/100050) (Ling et al. 2013) genomic database. After alignment, conserved primers TaMYB-7A-F and TaMYB-7A-R, covering the start and stop codon, were designed to isolate TaMYB-Al on the short arm of chromosome 7A.

Polymerase Chain Reaction (PCR) amplifications were conducted using high-fidelity Phusion DNA polymerase (Thermo-Fisher Scientific, Shanghai, China) in the Gene Amp PCR System 9700 (Thermo-Fisher Scientific, Shanghai, China) employing the following procedure: $2 \mathrm{~min}$ of denaturation at $98{ }^{\circ} \mathrm{C} ; 35$ cycles of $15 \mathrm{~s}$ at $98{ }^{\circ} \mathrm{C}, 30 \mathrm{~s}$ at $61{ }^{\circ} \mathrm{C}$, and $30 \mathrm{~s}$ at $72{ }^{\circ} \mathrm{C}$; and a final extension of $5 \mathrm{~min}$ at $72{ }^{\circ} \mathrm{C}$. The PCR products were extracted from a $1.0 \%$ agarose gel using the TIAN gel Midi Purification Kit (Tiangen Company)

*Further details about the Electronic Supplementary Material (ESM) can be found at the end of the article. 
and were cloned into the pGEM-T Easy Vector plasmid (Promega Corporation, Madison, WI, USA). The recombinant plasmid was then transformed into DH5 $\alpha$ Escherichia coli cells, and 10 positive clones were sent to a commercial company (Huada, Shenzheng, China) for sequencing. All of the primers used in this study are listed in Table S2. The primers Tamyb-7A attB1 forward /Tamyb-7A attB2 reverse and attB1 adapter / attB2 adapter were used to get the transient expression constructs pBRACT214-TaMYB-A1 and pBRACT214-TaMYB-a1.

\section{Bioinformatics analysis}

Blastx software was employed for searching the homologous sequences with an E-value of less than 1e-5 (https://urgi.versailles.inra.fr/blast/blast.php). The sequence alignments were conducted using Vector NTI 10 software (Thermo-Fisher Scientific, Shanghai, China). The primers were designed using Primer5 software (Premier Biosoft, Palo Alto, CA, USA). The phylogenetic trees of the MYB proteins were constructed using MEGA 4.0 (Tamura et al. 2007). The gene structures were drawn using the Gene Structure Display Server (http://gsds.cbi.pku.edu.cn/) (Guo et al. 2007).

\section{Transient expression}

The pBRACT214 transient vector (John Institute Centre, Norwich, UK), which contains the maize ubiquitin promoter, was used for the transient expression assay. The transient plasmids were constructed using the Gateway Cloning Kit (Thermo-Fisher Scientific, Shanghai, China). Five plasmids were obtained: pBRACT214-TaMYB-D1, pBRACT214TaMYB-A1, pBRACT214-TaMYB-a1, pBRACT214-ZmR (bHLH gene in maize), and pBRACT-control. pBRACT214-TaMYB-A1 and pBRACT214-TaMYB-D1 were mixed with pBRACT214-ZmR for transient expression experiments (Ahmed et al. 2003). The plasmids were delivered into the coleoptiles of 'Opata' by particle bombardment according to the methods of Ahmed et al. (2003). All of the treated coleoptiles were observed and photographed using a stereoscope (Leica Co., Wetzlar, Germany). The red cells were counted, and the cell numbers were calculated using Microsoft Excel 2003.

\section{Results}

The phenotypes of coleoptile colour in T. urartu, T. monococcum and T. aestivum

To understand the coleoptile colour phenotype more comprehensively, the seeds of 236 T. urartu cultivars, 14 T. monococcum cultivars, and 66 common wheat cultivars were germinated under standardized conditions, and their coleoptile colours were recorded. PI662236 was the only cultivar with uncoloured coleoptiles from the 236 T. urartu cultivars (Fig. 1A; Table S1), and all 14 T. monococcum cultivars had red coleoptiles (Table $\mathrm{S} 1)$. In contrast, there were only four cultivars that had the red grain trait out of the 66 common wheat cultivars planted widely at some time in the past in Qinghai Province (Table S1). The red coleoptile trait should be advantageous for T. urartu and T. monococ- 


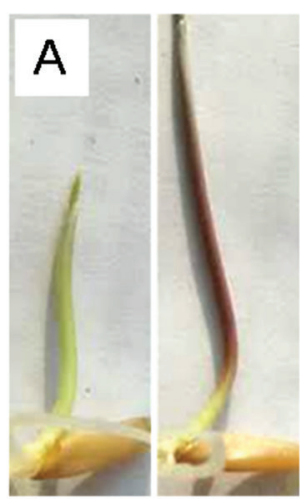

PI662236 PI662223

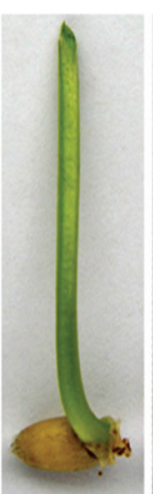

Opata

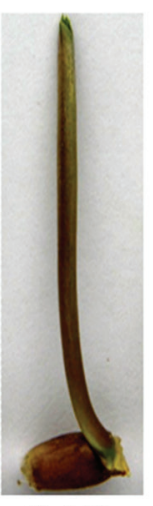

Gy115

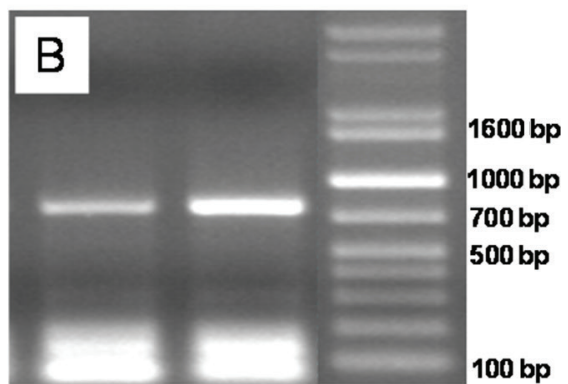

Gy115 PI662223 Marker

Figure 1. A, The coleoptile colour of Triticum urartu cultivars 'PI662236', 'PI662223', and bread wheat cultivars 'GY115' and 'Opata'. B, Amplification products using the conserved primers TaMYB-7A-F and TaMYB7A-R with cDNA templates from the coleoptiles of 'Gy115' and 'PI662223'3d after germination

cum cultivars surviving under natural conditions, while it had some disadvantage for the growth of $T$. aestivum cultivars under artificial conditions.

TaMYB-A1 isolation and its allelic variation in T. urartu and T. aestivum cultivars were sequenced and their accession numbers were listed in Table S1. Nucleotide sequences of all of the T. urartu TuMYB-A1s were similar to wild type TaMYB-A1. The whole genome sequence of TaMYB-A1 [KX373491] from 'Moyin2' was 867 bp and had an intron of $93 \mathrm{bp}$. The coding sequence of $T a M Y B-A 1$ was $774 \mathrm{bp}$, and encoded a protein of 258 amino acids (Fig. 1B). Four common wheat cultivars, 'Moyin2', 'Qingnong524', 'Gy584', and 'Gy115', had red coleoptiles, and four common wheat cultivars, 'Opata', 'Qingchun415', 'CS' and 'PI537976' had uncoloured coleoptiles. One single nucleotide ' $\mathrm{C}$ ' was deleted at $715 \mathrm{bp}$ after the start codon in all of the TaMYB-A1s isolated from the cultivars having uncoloured coleoptiles (Fig. S1). The allele was named TaMYB-a1 [KX373492]. The deletion caused a frame shift mutation, resulting in different amino acids after residue 239 when compared with the wild allele of TaMYB-A1. This region belonged to a regulatory activator domain, which was very important to MYBs ability to regulate the structural genes related to anthocyanin biosynthesis. TaMYB-al was also isolated from the cultivar ' $G Y 115$ ', while TaMYB-A1 existed in the other cultivars having purple coleoptiles (Table S1).

\section{The characteristics of TaMYB-A1}

TaMYB-A1 possessed the same intron-exon structure with one intron as TaMYB3 and $T a M Y B-D 1$, which regulate anthocyanin biosynthesis in common wheat, while the $M Y B$ genes in other plant species had two introns (Fig. 2A). TaMYB3, TaMYB-A1 and TaMYB$D 1$ had the same intron phase 1 as the first introns of the other MYBs. TaMYB-A1 has 258 amino acids, which is 15 amino acids shorter than $\mathrm{ZmCl}$ and six amino acids longer than 
A

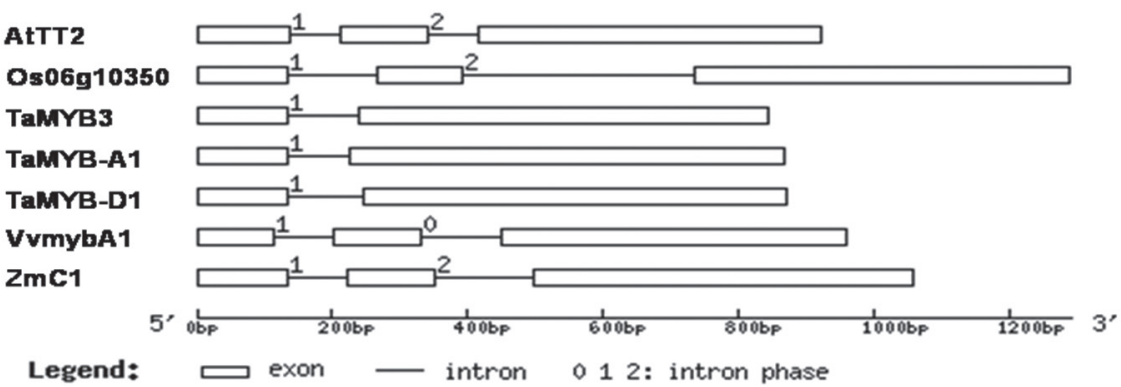

B

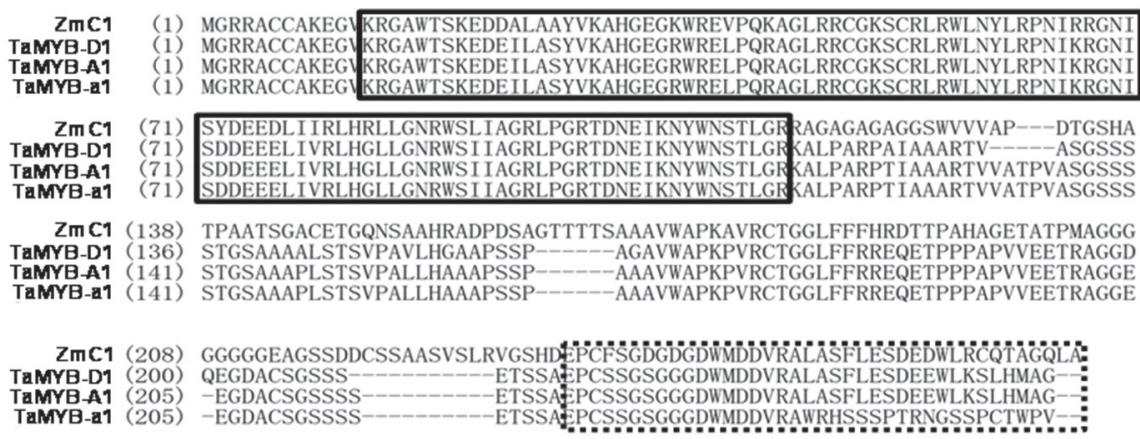

R2R3 Myb domain

:... Transcript activator domain

Figure 2. A, Gene structure of TaMYB-A1 and other $M Y B$ s regulating anthocyanin biosynthesis. B, Sequence alignment of the proteins ZmC1, TaMYB-D1, TaMYB-A1, and TaMYB-a1. The solid frame represents the MYB protein binding domain, and the dotted frame represents the regulatory activator domain. Accession numbers: VvmybA1, AB111101; ZmC1, AF320613; AtTT2, NC_003076; TaMYB3, KX455839; TaMYB-A1, KX373491; TaMYB-a1, KX373492; TaMYB-D1, KP136432

TaMYB-D1. TaMYB-A1 contains an integrated DNA-binding domain of 102 amino acids and a transcriptional domain of 42 amino acids, which is the same as in TaMYB-D1 (Fig. 2B). TaMYB-a1 corresponds to TaMYB-A1 over the first 238 amino acids, while the remaining 19 amino acids are different from those of TaMYB-A1. The non-corresponding amino acids are located in the transcriptional activator domain, which is important for the function of MYBs regulating anthocyanin biosynthesis.

\section{Phylogenetic tree of MYB proteins regulating anthocyanin biosynthesis}

The MYBs, including the genes regulating anthocyanin, were downloaded from NCBI to clarify the phylogenetic position of TaMYB-A1. TaMYB-A1 was assigned to the same branch of the phylogenetic tree as the other transcriptional factors regulating anthocyanin or flavonol synthesis (AtMYB12, AtTT2, ZmC1, TaMYB10-A, TaMYB10-B, TaMYB10D, TaMYB3, TaMYB-D1, and TaMYB320, Fig. 3). 


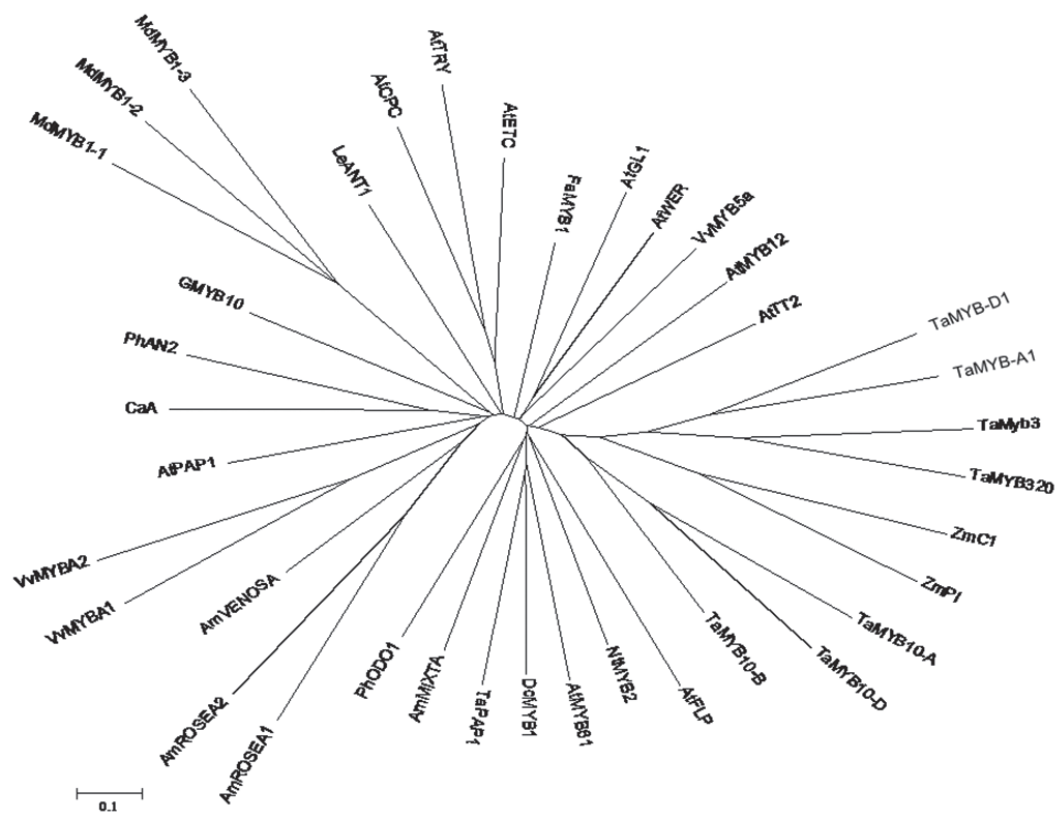

Figure 3. Phylogenetic tree of the MYB proteins based on MYB domains. Accession numbers: AmMIXTA, CAA55725; AmROSEA1, ABB83826; AmROSEA2, ABB83827; AmVENOSA, ABB83828; AtCPC, NP_182164; AtETC, NP_171645; AtFLP, NP_563948; AtGL1, AAC97387; AtMYB12, ABB03913; AtMYB61, NP_172425; AtPAP1, ABB03879; AtTRY, NP 200132; AtTT2, Q9FJA2; AtWER, AAF18939; CaA, CAE75745; DcMYB1, BAE54312; FaMYB1, AAK84064; GMYB10, CAD87010; LeANT1, AAQ55181; MdMYB1-1, ABK58136; MdMYB1-2, ABK58137; MdMYB1-3, ABK58138; NtMYB2, BAA88222; PhAN2, AAF66727; PhODO1, AAV98200; VvMYB5a, AAS68190; VvMYBA1, BAD18977; VvMYBA2, BAD18978; ZmC1, AAA33482; ZmPl, AAA19821; TaMYB-D1, KP136432; TaMYB-A1,KX373491; TaMYB10-A, AB599721; TaMYB10-B, AB599722; TaMYB10-D, AB191460; TaMyb3, KX455839; TaMYB320, AB252146

\section{Functional verification of TaMYB-A1}

TaMYB-A1 was located closest to TaMYB-D1 in the phylogenetic tree, and TaMYB-A1 also had the same DNA-binding domain and transcriptional domain as TaMYB-D1. $T a M Y B-D 1$ has a regulatory function improving anthocyanin biosynthesis (Wang et al. 2015). Thus, TaMYB-D1 was selected as the positive control for evaluating the function of TaMYB-A1. In the present experiment, the coding sequences of $Z m R$, TaMYB-al, TaMYB-A1, and TaMYB-D1 were placed after the ubiquitin promoter in the pBRACT214 vector. The transient expression levels of TaMYB-A1 and TaMYB-D1 induced anthocyanin biosynthesis in the coleoptile cells of 'Opata' with the assistance of ZmR, while TaMYB-al did not induce anthocyanin biosynthesis. Only TaMYB-a1, TaMYB-A1, and TaMYB-D1 yielded no red cells in the coleoptiles (Fig. 4). This meant that TaMYB-a1 was a loss-of-function mutant, while TaMYB-A1 could regulate anthocyanin biosynthesis. 


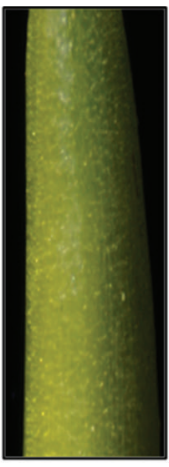

CK

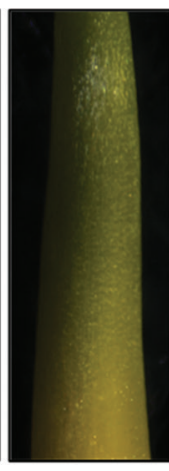

$\mathbf{Z m R}$

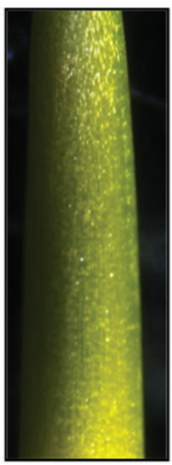

TaMYB-a1

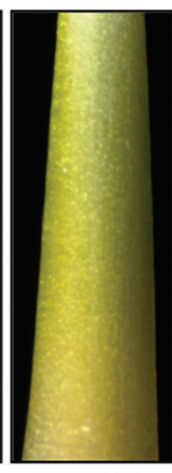

TaMYB-A1
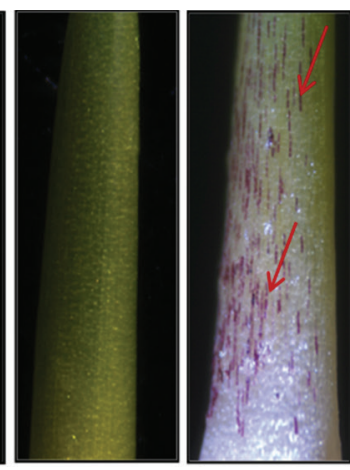

ZmR+TaMYB-a1 ZmR+TaMYB-A1 ZmR+TaMYB-D1

Figure 4. Coleoptiles independently bombarded with six plasmids. CK indicates the transformation with the empty vector pBRACT214, while TaMYB-a1, TaMYB-A1, TaMYB-D1, and ZmR represent transformations with pBRACT214-TaMYB-a1, pBRACT214-TaMYB-A1, pBRACT214-TaMYB-D1, and pBRACT214-ZmR, respectively. Red arrows indicate cells accumulating anthocyanin

\section{Discussion}

Coleoptile colour trait was an important trait relative to the adaptation of $\mathrm{T}$. urartu and $\mathrm{T}$. aestivum

All accessions of T. urartu were collected from Middle East in this manuscript (Table S1). In Middle East, T. urartu faces drought, hotness and strong light frequently at the germination stage. Red coleoptile can provide exceptional protection against these stresses compared with uncoloured coleoptile (Ahmed et al. 2015; de Oliveira et al. 2015; Ilk et al. 2015). Consequently, all accessions of T. urartu had red coleoptiles except one accession from Iran. The above-mentioned stresses occur rarely in the cultivated field of Qinghai province, and red coleoptile should have no more advantage over uncoloured coleoptile in this condition. Moreover, red coleoptile $(R c-1)$ and the genes for purple grain $(P p-1)$ are close linked on the chromosomes of homoeologous group 7 (Himi et al. 2005; Tereshchenko et al. 2012), and purple grain can influence flour colour, which reduce the visual and taste sensation of flour (Wende et al. 2007). Selection against purple grain leads to co-selection against red coleoptile. As a consequence, uncoloured coleoptile was dominant in T. aestivum cultivars from Qinghai province, China. At all, coleoptile colour trait was an important trait relative to the adaptation of T. urartu and T. aestivum.

\section{TaMYB-A1 was the MYB transcription factor regulating anthocyanin biosynthesis}

$T a M Y B-A 1$ was isolated based on the nucleotide sequence of TaMYB-D1, belonged in the phylogenetic tree to the group of MYBs regulating anthocyanin biosynthesis (Fig. 3). AtMYB12 regulates caffeoylquinic acid and flavonol synthesis in tobacco and tomato (Luo et al. 2008) and also induces anthocyanin biosynthesis in wheat coleoptiles (Gao et al. 2011). AtTT2 is the key gene for the biosynthesis of pro-anthocyanidins in the testes of 
Arabidopsis (Nesi et al. 2001). TaMYB10-A, TaMYB10-B, and TaMYB10-D are related to red grain colour in wheat (Himi and Noda 2005). $\mathrm{ZmCl}$ was the first $M Y B$ gene regulating anthocyanin biosynthesis to be isolated from maize (Cone et al. 1986). $\mathrm{ZmCl}$ possesses special DNA-binding and transcriptional domains for promoting the expression of the structural genes involved in anthocyanin biosynthesis (Grotewold et al. 2000), and $\mathrm{Ta}$ $M Y B-D 1$ is a candidate gene for $R c-D 1$ (Wang et al. 2015). TaMYB-A1 had the same DNA-binding and regulatory activator domains as TaMYB-D1 and $\mathrm{ZmC1}$, which indicated that it may up-regulate the structural genes of anthocyanin biosynthesis. Moreover, TaMYB-A1 could induce anthocyanin biosynthesis in the coleoptile cells of the common wheat cultivar 'Opata' with the help of the bHLH transcription factor ZmR. Therefore, TaMYB-A1 should be the $M Y B$ gene responsible for anthocyanin biosynthesis in wheat.

TaMYB-A1 was the candidate gene for Rc-A1

$T a M Y B-A 1$ was identical to the TaC1-1 homolog TaC1-A1 suggested to be candidate genes for $R c-1$ (Himi and Taketa 2015), but the function of TaC1-A1 and its distribution in natural population was unclear. In this manuscript, transient function verification and allelic variation in natural population were employed for further certifying its role in controlling red coleoptile trait. The common wheat cultivars with uncoloured coleoptiles in our experiment had the allele TaMYB-a1, which contains a single nucleotide ' $C$ ' deleted $715 \mathrm{bp}$ after the start codon. The regulatory activator domain of TaMYB-al had 19 amino acids that were different from those of the wild type TaMYB-A1, which are very important for MYBs ability to regulate anthocyanin biosynthesis. TaMYB-a1 could not induce anthocyanin biosynthesis in the coleoptiles cells of the common wheat cultivars, resulting in uncoloured coleoptiles. All of the common wheat cultivars having red coleoptiles carried TaMYB-A1, except 'Gy115'. The red coleoptiles 'Gy115' has been proved to be controlled by one dominant gene TaMYB-D1 (Wang et al. 2015). Therefore, TaMYB-al should not influence the red coleoptile trait in 'Gy115'. All 34 of the selected T. urartu cultivars with red coleoptiles carried TuMYB-A1 identical to TaMYB-A1. Moreover, $R c-1$ and TaMyb-1 co-locatizate exactly on the short arms of the homoeologous group 7 chromosomes (Himi and Taketa 2015; Wang et al. 2015). All evidences strongly suggest that $T a M Y B-A 1$ was the $R c-A 1$ candidate responsible for red coleoptiles.

\section{Accession numbers}

Nucleotide sequences of the TuMYB-A1 and TaMYB-A1 genes isolated in the current study were deposited in Gen Bank under accession numbers KX373488-KX373530.

\section{Acknowledgements}

This research was financially supported by the Basic Research Projects of Qinghai Province (2015-ZJ-701), the Pilot Projects of Designer Breeding by Molecular Modules, Chinese Academy of Sciences, the National Natural Science Foundation of China 
(no. 31071417, 31260322) and the West Light Foundation of the Chinese Academy of Sciences.

\section{References}

Ahmed, N., Maekawa, M., Utsugi, S., Himi, E., Ablet, H., Rikiishi, K., Noda, K. 2003. Transient expression of anthocyanin in developing wheat coleoptile by maize $C 1$ and B-peru regulatory genes for anthocyanin synthesis. Breed. Sci. 53:29-34.

Ahmed, N., Maekawa, M., Utsugi, S., Rikiishia, K., Ahmad, A., Noda, K. 2006. The wheat $R c$ gene for red coleoptile colour codes for a transcriptional activator of late anthocyanin biosynthesis genes. J. Cereal Sci. 44:54-58.

Ahmed, N.U., Park, J.I., Jung, H.J., Hur, Y., Nou, I.S. 2015. Anthocyanin biosynthesis for cold and freezing stress tolerance and desirable colour in Brassica rapa. Funct. Integr. Genomics 15:383-394.

Bleiss, W., Ludwig, M. 1990. Rapid growth-responses of dark-grown wheat seedlings to red-light irradiation .2. Kinetic-studies on the growth of different coleoptile zones. Physiol. Plant. 80:205-209.

Borevitz, J.O., Xia, Y., Blount, J., Dixon, R.A., Lamb, C. 2000. Activation tagging identifies a conserved MYB regulator of phenylpropanoid biosynthesis. Plant Cell 12:2383-2394.

Cone, K.C., Burr, F.A., Burr, B. 1986. Molecular analysis of the maize anthocyanin regulatory locus C1. Proc. Natl Acad. Sci. USA 83:9631-9635.

de Oliveira, A.F., Mercenaro, L., Del Caro, A., Pretti, L., Nieddu, G. 2015. Distinctive anthocyanin accumulation responses to temperature and natural UV radiation of two field-grown Vitis vinifera L. cultivars. Molecules 20:2061-2080.

Gao, X., Zhang, L., Zhou, S., Wang, C., Deng, X., Zhang, H., Yang, G., Javeed, H., He, G. 2011. AtMYB12 gene: a novel visible marker for wheat transformation. Mol. Biol. Rep. 38:183-190.

Gonzali, S., Mazzucato, A., Perata, P. 2009. Purple as a tomato: towards high anthocyanin tomatoes. Trends Plant Sci. 14:237-241.

Grotewold, E., Sainz, M.B., Tagliani, L., Hernandez, J.M., Bowen, B., Chandler, V.L. 2000. Identification of the residues in the Myb domain of maize $\mathrm{C} 1$ that specify the interaction with the bHLH cofactor R. Proc Natl Acad. Sci. USA 97:13579-13584.

Guo, A.Y., Zhu, Q.H., Chen, X., Luo, J.C. 2007. GSDS: a gene structure display server. Yi Chuan. 29:10231026.

Himi, E., Nisar, A., Noda, K. 2005. Colour genes (R and Rc) for grain and coleoptile upregulate flavonoid biosynthesis genes in wheat. Genome 48:747-754.

Himi, E., Noda, K. 2005. Red grain colour gene (R) of wheat is a Myb-type transcription factor. Euphytica. 143:239-242.

Himi, E., Maekawa, M., Miura, H., Noda, K. 2011a. Development of PCR markers for Tamyb10 related to R-1, red grain color gene in wheat. Theor. Appl. Genet. 122:1561-1576.

Himi, E., Maekawa, M., Noda, K. 2011b. Differential expression of three flavanone 3-hydroxylase genes in grains and coleoptiles of wheat. Int. J. Plant Genomics. 2011:369460.

Himi, E., Taketa, S. 2015. Isolation of candidate genes for the barley Ant1 and wheat Rc genes controlling anthocyanin pigmentation in different vegetative tissues. Mol. Genet. Genomics 290:1287-1298.

Ilk, N., Ding, J., Ihnatowicz, A., Koornneef, M., Reymond, M. 2015. Natural variation for anthocyanin accumulation under high-light and low-temperature stress is attributable to the ENHANCER OF AG-4 2 (HUA2) locus in combination with PRODUCTION OF ANTHOCYANIN PIGMENT1 (PAP1) and PAP2. New Phytol. 206:422-435.

International Wheat Genome Sequencing Consortium 2014. A chromosome-based draft sequence of the hexaploid bread wheat (Triticum aestivum) genome. Science 345:286-299.

Kayesh, E., Shangguan, L.F., Korir, N.K., Sun, X., Bilkish, N., Zhang, Y.P., Han, J., Song, C.N., Cheng, Z.M., Fang, J.G. 2013. Fruit skin color and the role of anthocyanin. Acta Physiol. Plant. 35:2879-2890. 
Khlestkina, E.K., Pestsova, E.G., Röder, M.S., Börner, A. 2002. Molecular mapping, phenotypic expression and geographical distribution of genes determining anthocyanin pigmentation of coleoptiles in wheat (Triticum aestivum L.). Theor. Appl. Genet. 104: 632-637.

Khlestkina, E.K., Röder, M.S., Salina, E.A. 2008. Relationship between homoeologous regulatory and structural genes in allopolyploid genome - a case study in bread wheat. BMC Plant Biol. 8:88.

Lawson, V.R., Weintraub, R.L. 1975. Interactions of microtubule disorganizers, plant hormones, and red-light in wheat coleoptile segment growth. Plant Physiol. 55:1062-1066.

Ling, H.Q., Zhao, S.C., Liu, D.C., Wang, J.Y., Sun, H., Zhang, C., Fan, H.J., Li, D., Dong, L.L., Tao, Y., Gao, C., Wu, H.L., Li, Y.W., Cui, Y., Guo, X.S., Zheng, S.S., Wang, B., Yu, K., Liang, Q.S., Yang, W.L., Lou, X.Y., Chen, J., Feng, M.J., Jian, J.B., Zhang, X.F., Luo, G.B., Jiang, Y., Liu, J.J., Wang, Z.B., Sha, Y.H., Zhang, B.R., Wu, H.J., Tang, D.Z., Shen, Q.H., Xue, P.Y., Zou, S.H., Wang, X.J., Liu, X., Wang, F.M., Yang, Y.P., An, X.L., Dong, Z.Y., Zhang, K.P., Zhang, X.Q., Luo, M.C., Dvorak, J., Tong, Y.P., Wang, J., Yang, H.M., Li, Z.S., Wang, D.W., Zhang, A.M., Wang, J. 2013. Draft genome of the wheat A-genome progenitor Triticum urartu. Nature 496:87-90.

Luo, J., Butelli, E., Hill, L., Parr, A., Niggeweg, R., Bailey, P., Weisshaar, B., Martin, C. 2008. AtMYB12 regulates caffeoyl quinic acid and flavonol synthesis in tomato: expression in fruit results in very high levels of both types of polyphenol. Plant J. 56:316-326.

Mano, H., Ogasawara, F., Sato, K., Higo, H., Minobe, Y. 2007. Isolation of a regulatory gene of anthocyanin biosynthesis in tuberous roots of purple-fleshed sweet potato. Plant Physiol. 143:1252-1268.

McClintock, B. 1950. The origin and behavior of mutable loci in maize. Proc. Natl Acad. Sci. USA 36:344-355.

Nesi, N., Jond, C., Debeaujon, I., Caboche, M., Lepiniec, L. 2001. The Arabidopsis TT2 gene encodes an R2R3 MYB domain protein that acts as a key determinant for proanthocyanidin accumulation in developing seed. Plant Cell 13:2099-2114.

Pourcel, L., Bohorquez-Restrepo, A., Irani, N.G., Grotewold, E. 2012. Anthocyanin biosynthesis, regulation, and transport: new insights from model species. Recent Advances in Polyphenol Research 3:143-160.

Quattrocchio, F., Wing, J., van der Woude, K., Souer, E., de Vetten, N., Mol, J., Koes, R. 1999. Molecular analysis of the anthocyanin 2 gene of petunia and its role in the evolution of flower colour. Plant Cell 11:1433-1444.

Tamura, K., Dudley, J., Nei, M., Kumar, S. 2007. MEGA4: Molecular evolutionary genetics analysis (MEGA) software version 4.0. Mol. Biol. Evol. 24:1596-1599.

Tereshchenko, O.Y., Gordeeva, E.I., Börner, A., Khlestkina, E.K., Arbuzova, V.S. 2012. The D genome carries a gene determining purple grain colour in wheat. Cereal Res. Commun. 40:334-341.

Wang, Y.Q., Hou, X.J., Zhang, B., Chen, W.J., Liu, D.C., Liu, B.L., Zhang, H.G. 2015. Identification of a candidate gene for Rc-D1, a locus controlling red coleoptile colour in wheat. Cereal Res. Commun. 43:1-12.

Wende, L., Pickard, M.D., Trust, B. 2007. Evaluation of antioxidant activity and electronic taste and aroma properties of antho-beers from purple wheat grain. J. Agric. Food Chem. 55:8958-8966.

Xie, R.J., Zheng, L., He, S.L., Zheng, Y.Q., Yi, S.L., Deng, L. 2011. Anthocyanin biosynthesis in fruit tree crops: Genes and their regulation. Afr. J. Biotechnol. 10:19890-19897.

Yan, Z.H., Wan, Y.F., Liu, K.F., Zheng, Y.L., Wang, D.W. 2002. Identification of a novel HMW glutenin subunit and comparison of its amino acid sequence with those of homologous subunits. Chin. Sci. Bull. 47:220-225.

Zhang, F., Wan, X.Q., Zheng, Y.X., Sun, L.X., Chen, Q.B., Guo, Y.L., Zhu, X.Q., Liu, M. 2014. Physiological and related anthocyanin biosynthesis genes responses induced by cadmium stress in a new coloured-leaf plant "Quanhong Poplar". Agroforestry Syst. 88:343-355.

Zhang, Y., Butelli, E., Destefano, R., Schoonbeek, H.J., Magusin, A., Pagliarani, C., Wellner, N., Hill, L., Orzaez, D., Granell, A. 2013. Anthocyanins double the shelf life of tomatoes by delaying overripening and reducing susceptibility to gray mold. Cur. Bio. 23:1094-1100. 


\section{Electronic Supplementary Material (ESM)}

Electronic Supplementary Material (ESM) associated with this article can be found at the website of CRC at http://www.akademiai.com/content/120427/

Electronic Supplementary Table S1.

Information about the cultivars involved in the research work

Electronic Supplementary Table S2.

Primers used in the research work

Electronic Supplementary Figure S1. The sequence alignment of TaMYB-a1 [KX373492] and TaMYB-A1 [KX373491]. The solid frame represents the deletion of ' $\mathrm{C}$ ' 